\title{
LncRNA H19 promotes the differentiation of bovine skeletal muscle satellite cells by suppressing Sirt1/FoxO1
}

\author{
Xiaochun $\mathrm{Xu}^{1+}$, Shengyue $\mathrm{Ji}^{2+}$, Weili $\mathrm{Li}^{2}$, Bao $\mathrm{Yi}^{2}$, Hengxin $\mathrm{Li}^{2}$, Hongfu Zhang ${ }^{2^{*}}$ and Wenping Ma ${ }^{{ }^{*}}$
}

\begin{tabular}{l}
\hline *Correspondence: \\
wt1505@126.com; \\
petermama@163.com \\
'Equal contributors \\
2State Key Laboratory of Animal \\
Nutrition, Institute of Animal \\
Science, Chinese Academy of \\
Agricultural Sciences, No. 2 \\
Yuanmingyuan West Road, Beijing \\
100193, People's Republic of China \\
'College of Biology Sciences and \\
Engineering, Beifang University of \\
Nationalities, Yinchuan, Ningxia, \\
People's Republic of China
\end{tabular}

* Correspondence: wt1505@126.com

${ }^{\dagger}$ Equal contributors

${ }^{2}$ State Key Laboratory of Animal Nutrition, Institute of Animal Science, Chinese Academy of Yuanmingyuan West Road, Beijing 100193, People's Republic of China Colege of Biology Sciences and People's Republic of China

\begin{abstract}
Background: $\mathrm{H} 19$ is a well-characterized Long noncoding RNA (IncRNA) that has been proven to promote myoblast differentiation in humans and mice. However, its mechanism of action is still not fully interpreted.

Methods: Using RT-qPCR, we examined H19 RNA levels in various tissues from 1-week, 1-month, 6-month and 36-month old male cattle (i.e., newborn, infant, young and adult). The protein and mRNA levels of MyoG, MyHC, Sirt1 and FoxO1 in the satellite and $\mathrm{C}_{2} \mathrm{C}_{12}$ cells with an $\mathrm{H} 19$ silencing or overexpression vector were respectively detected using western blot and real-time qPCR.

Results: H19 was highly expressed in skeletal muscle at all the studied ages. High expression of H19 was required for the differentiation of bovine satellite cells. Knockdown of $\mathrm{H} 19$ caused a remarkable increase in the myoblast-inhibitory genes Sirt1/FoxO1, suggesting that H19 suppresses Sirt1/FoxO1 expression during myogenesis. Western blotting analysis of co-transfection of Sirt1 or FoxO1 expression vectors with pcDNA-H19 indicated that Sirt1/FoxO1 overexpression neutralized the promotion of myoblast differentiation through transfection of pcDNA-H19.
\end{abstract}

Conclusion: $\mathrm{H} 19$ promoted the differentiation of bovine skeletal muscle satellite cells by suppressing Sirt1/FoxO1.

Keywords: H19 IncRNA, Myoblast differentiation, Sirt1, FoxO1

\section{Introduction}

Skeletal myogenesis is a complex process that depends on the modulation of a series of genes. Myogenic differentiation antigen (MyoD) and myogenic factor 5 (Myf5) enable the differentiation of myogenic progenitors into myoblasts, and then myogenin (MyoG or Myf4) promotes the myoblasts to differentiate into myotubes [1, 2]. Several other factors negatively regulate the differentiation process, including forkhead proteins (FoxO), which inhibit myogenic differentiation by stabilizing Notch/Hes binding. In the absence of FoxO1, MyoD expression is elevated and myogenic differentiation is enhanced [3].

Long noncoding RNAs (lncRNAs) have recently emerged as an important class of gene expression regulators. They are localized both in the nucleus and the cytosol, and play an important role in gene expression regulation at every stage of life $[4,5]$. The contributions of lncRNAs have thus far mostly been investigated in cancer and 
neurological disorders, while the role of lncRNA in myogenesis is still poorly reported. Only a few lncRNAs were proven to be involved in myogenic differentiation. For instance, linc-MD1 is a muscle-specific intergenic lncRNA that acts as a sponge for miR-133 and miR-135, preventing their suppression of MAML1 and MEF2C and activating muscle-specific gene expression [6]. These discoveries illustrate that lncRNAs have greater potential that remain to be discovered and characterized.

H19 is a well-characterized lncRNA in mammalian cells. It is highly expressed in human and mouse developing embryos and adult skeletal muscles [7] and it is upregulated during myoblast differentiation and muscle regeneration [8]. H19 functions as a post-transcriptional suppressor or miRNA sponge for let-7, helping to regulate the Igf2 signaling pathway $[9,10]$. It also encodes the conserved microRNAs miR-675-3p and $-5 \mathrm{p}$, which target Smad1, Smad5 and Cdc6, and thus promotes skeletal muscle differentiation and regeneration [8].

Muscle satellite cells are a heterogeneous population of committed myogenic progenitors and non-committed stem cells located beneath the basal lamina of skeletal muscle fibers [11, 12]. Described as quiescent myoblasts, they are crucial for the repair of muscle injury $[13,14]$. The isolation, culture, and regulation of differentiation of muscle satellite cells are important methods for muscle biological research.

Here, we report that H19 was highly expressed in skeletal muscle in tissues from cattle of all postnatal ages. Knockdown of H19 seriously hindered the differentiation of skeletal muscle satellite cells. Gene expression analysis after H19 silencing or overexpression revealed that its promoting effect might be associated with blocking of the Sirt1/FoxO1 signaling pathway. Our findings revealed a novel pathway of H19 in myogenesis regulation.

\section{Materials and methods}

Ethics statement

All the experimental procedures involving cattle were approved by the Institute of Animal Science, Chinese Academy of Agricultural Sciences. The cattle were monitored in a stress-free environment where they were given food and water ad libitum in a humidity- and temperature-controlled room in the Experimental Farm of the Institute of Animal Science in Beijing.

\section{Samples}

The heart, intestinal smooth muscle (smth-muscle), tibia skeletal muscle (sk-muscle), liver, spleen, kidney and lung were sampled from six examples each of 1-week (1w, representing newborns), 1-month (1 $\mathrm{m}$, representing infants), 6-month $(6 \mathrm{~m}$, representing young animals) and 36-month old male cattle (36 m, representing well-developed adults). Embryo samples were obtained from three cows that were one month pregnant (E30), i.e., at a moment when the fetus has not yet formed. The samples for each age were mixed for further experimental analysis, and all procedures were performed in triplicate.

\section{Expression profile of $\mathrm{H} 19$ in various tissues from cattle of different ages}

Total RNA was isolated from the various tissue and embryo samples. A TaKaRa Reverse Transcription Kit was used to obtain the first chain cDNA. Subsequent real- 
time qPCR reactions were carried out in a final volume of $25 \mu \mathrm{l}$, using SYBR Premix Ex Taq (TaKaRa), $0.4 \mathrm{mM}$ of primer and $200 \mathrm{ng}$ of cDNA template. Each individual sample was run in triplicate wells. The reactions were initially denatured at $95{ }^{\circ} \mathrm{C}$ for $3 \mathrm{~min}$ followed by 35 cycles of $95{ }^{\circ} \mathrm{C}$ for $15 \mathrm{~s}$ and $60{ }^{\circ} \mathrm{C}$ for $1 \mathrm{~min}$. Amplicon quantification was performed using ABI 7300 software (Applied Biosystem). The primers used in the reactions were: H19 (F: 5'-TTC CCA GCC GCC ACT TC-3', R: 5'-GAG CCG CTC CTG TGACCT ACT-3'), 18S RNA (F: 5'-GAGAAACGGCTACCACATCC-3', R :5' GCCAGACTTGCCCTCCA-3'), and GAPDH RNA (F: 5'-GCAAGTTCAACGGCACAG-3', R: 5'-CGCCAGTAGACTCCACGACAT-3'). The change in transcript abundance of all tested genes was calculated using the $2^{-\Delta \Delta} \mathrm{Ct}$ method. All mRNA amounts were normalized to the GAPDH or 18S RNA control.

\section{Generation of constructs}

The pLenti-H19, pLenti-NTC (the vector without the H19 gene), and pcDNA-H19 (the overexpression vector of $\mathrm{H} 19$ gene) interference plasmids were effectively constructed and identified by Dingguo Changsheng Biotechnology Co. LTD., according to the manufacturer's instruction. The pcDNA-Sirt1 and pcDNA-FoxO1 were donated by Professor Shihuan Kuang (Purdue University).

The full-length bovine $\mathrm{H} 19$ gene was amplified with the sequence released in Genbank (Accession number: AC_000186.1) as the template. Primers applied were: F: 5'-CGG GGT ACC TGC GTG GGA GGG TGA AAG A-3', R: 5'-CCC AAG CTT GGA GGG ACA CTA GGC AAG ATG G-3'. The product was cleaved and ligated into the corresponding sites of pcDNA-3.1 plasmid and verified via sequencing.

\section{Cell culture and transfection}

Satellite cells were isolated from three 36-month old healthy adult cattle using the previously reported method [15]. $\mathrm{C}_{2} \mathrm{C}_{12}$ cells were bought from Baili Biotechnology Co., Ltd. The satellite and $\mathrm{C}_{2} \mathrm{C}_{12}$ cells were transfered in growth medium consisting of DMEM/F12 (Invitrogen) with 10\% FBS, then incubated in a humidified incubator with an atmosphere of $95 \%$ air $-5 \% \mathrm{CO}_{2}$ at $37^{\circ} \mathrm{C}$.

When the cell confluence reached about 70\%, $6 \mu \mathrm{g}$ of pLenti-H19, pLenti-NTC (negative control) or pcDNA-H19 were transfected or co-transfected with expression vectors of pcDNA-Sirt1 or pcDNA-FoxO1 into the satellite cells and $\mathrm{C}_{2} \mathrm{C}_{12}$ cells with Lipofectamine 3000 (Invitrogen) according to the manufacturer's instructions. The cells were then induced to differentiate. After transfection for $96 \mathrm{~h}$, the expressions of MyoG, MyHC, Sirt1 and FoxO1 were detected using western blotting and RT-qPCR.

\section{Differentiation assay}

To induce myoblast differentiation, the growth medium was replaced with differentiation medium (DMEM supplemented with $2 \%$ horse serum) when cell confluence reached $80 \%$. Thereafter, the cultured cells were fixed with $3.7 \%$ formaldehyde, and then microscopically analyzed to determine the fraction of MHC-positive cells and the fusion indices. Permeabilization and blocking of non-specific binding were performed using phosphate buffer solution (PBS, pH 7.4) containing $0.1 \%$ Triton X-100 and $0.2 \%$ bovine serum albumin (BSA) for $1 \mathrm{~h}$. The cells were incubated with anti-MHC (1:50) for $1 \mathrm{~h}$ at room temperature, 
then washed three times with PBS containing 0.1\% Triton X-100. Next, they were incubated with secondary antibodies for $45 \mathrm{~min}$ at room temperature. After subsequent incubation of the cells with Hoechst 33342 for 10 min, they were washed three times, and Fluoromount G staining was applied. Fluorescence detection was performed using an Olympus IX51 inverted microscope. Random fields were analyzed with a 20X objective. The cell differentiation ratio was analyzed with Cellometer Cell Counters and Cell Analysis Systems.

\section{RNA extraction and real-time qPCR}

Total RNA was isolated from the satellite cells and $\mathrm{C}_{2} \mathrm{C}_{12}$ at the start and on each of the first 5 days of the differentiation induction. RT-PCR for H19 lncRNA was performed using TRIzol reagent (Invitrogen) following the manufacturer's instructions. Amplicon quantification was performed using a real-time qPCR protocol that was in accordance with the protocol for expression level detection of H19 in various tissues (mentioned above). The primers used in the reactions were: H19 (F: 5'-TTC CCA GCC GCC ACT TC-3', R: 5' -GAG CCG CTC CTG TGACCT ACT-3'); MyoG (F: 5' GAG GAA GTC TGT GTC GGT GG-3', R: 5'-CCA CGA TGG ACG TAA GGG AG3'); MyoHC (F: 5'-GCA TCC CTA AAG GCA GGC TC-3', R: 5'-GCC ACT TGT AGG GGT TGA CA-3'); Sirt1 (F: 5'-AGA ACC ACC AAA GCG GAA A-3', R: 5' TCC CAC AGG AGA CAG AAA CC-3'); Foxo1 (F: 5'-CCC AGG CCG GAG TTT AAC C-3', R: 5'-GTT GCT CAT AAA GTC GGT GCT-3'); GAPDH (F: 5'-GCA AGT TCA ACG GCA CAG-3', R: 5' -CGC CAG TAG ACT CCA CGA CAT-3'). The change in transcript abundance of all tested genes was calculated using the $2^{-\Delta \Delta} \mathrm{Ct}$ method. All mRNA amounts were normalized to the GAPDH control.

\section{Western blotting}

$25 \mu \mathrm{g}$ of protein isolated from each sample were separated using 12\% SDS-PAGE and electro-transferred to a PVDF membrane (Millipore) for immunoblot analysis. The following primary antibodies were used: anti-Sirt1 (Abcam, ab32441, 1:500), anti-FoxO1 (Abcam, ab39670, 1:500), anti-MyoG (Abcam, ab1835, 1:500), anti-MyHC (Abcam, ab15, 1:500) and anti-GAPDH (Santa Cruz, sc-166574, 1:800), which was used as the reference. After incubation with the appropriate HRP-conjugate secondary antibody, proteins were detected using a ChemiDoc XRS imaging system and analyzed using Quantity One software (Bio-Rad). The protein abundance was normalized to the GAPDH gene. pLenti-NTC was used as a negative control. The blank control was proteins of the satellite and $\mathrm{C}_{2} \mathrm{C}_{12}$ cells without any treatment, but cultivated for the same period. GAPDH was used as an internal control.

\section{Statistical analysis}

All data were presented as means \pm S.E.M. of three biological replicates. Two-tailed Student's $t$-test was employed to determine $\mathrm{p}$ values. The significance was set at $p<0.05(n=3)$.

\section{Results}

H19 was highly expressed in skeletal muscle in cattle of all ages

H19 RNA levels in various tissues of 1-week, 1-month, 6-month, and 36-month old male cattle were determined. The embryo was regarded as a positive control for H19, 
based on its high expression level at the embryo stage. To verify the accuracy of the results, GAPDH RNA (Fig. 1) and 18S RNA (Additional file 1: Figure S1) were used as reference genes. The results showed that the change patterns for H19 with 18S RNA and GAPDH RNA as reference genes were the same, indicating the high accuracy of the selected reference genes. Of the tissues we assessed, H19 was only highly expressed in skeletal muscle (sk-muscle) at all the ages. With age, H19 exhibited a moderate downward trend (Fig. 1, Additional file 1: Figure S1).

\section{H19 level rose during myoblast differentiation}

Satellite cells were obtained from skeletal muscle 36 -month old cattle. When $80 \%$ confluence was reached, the cells, including the $\mathrm{C}_{2} \mathrm{C}_{12}$ cells, were induced to differentiate through the addition of $2 \%$ horse serum in DMEM (Invitrogen). The expression levels of $\mathrm{H} 19$ during myoblast differentiation were determined via RT-qPCR. The results showed that the expression level of H19 RNA in bovine skeletal muscle satellite cells and $\mathrm{C}_{2} \mathrm{C}_{12}$ myoblasts increased with differentiation time (Fig. 2a and b).

\section{Silencing $\mathrm{H} 19$ suppressed myoblast differentiation of skeletal muscle satellite cells}

To explore the effect of $\mathrm{H} 19$ on myoblast differentiation, a pLenti-H19 interference vector was transfected into the satellite and $C_{2} C_{12}$ cells, and then the cells were induced to differentiate. After 4 days, the cells were observed with a microscope and their differentiation ratio was analyzed. The fluoromount $\mathrm{G}$ staining and silencing efficiency results showed that the H19 gene was silenced after transfection of pLenti-H19 (Fig. 3a and b). The differentiation ratios of bovine satellite and $\mathrm{C}_{2} \mathrm{C}_{12}$ cells were both decreased by silencing H19 (Fig. 3c). Consistent with this change, the protein expression level of the myoblast differentiation maker genes $M y o G$ and $M y H C$ were significantly downregulated in bovine satellite cells (Fig. $3 \mathrm{~d}-\mathrm{g}$ ). Interestingly, we found that Sirt1 and FoxO1 expressions were remarkably upregulated (Fig. 3c). These were previously reported as myoblast inhibitors. As per the protein level change pattern, mRNA levels of $M y o G$ and $M y o H C$ in pLentiNTC infected bovine sk-satellite and $\mathrm{C}_{2} \mathrm{C}_{12}$ cells were significantly lower than in pLentiH19 infected cells on day 4 after the induction of differentiation, while the mRNA levels of Sirt1 and FoxO1 had the opposite trend (Fig. $3 \mathrm{~h}-\mathrm{k}$ ). These data suggest that high levels

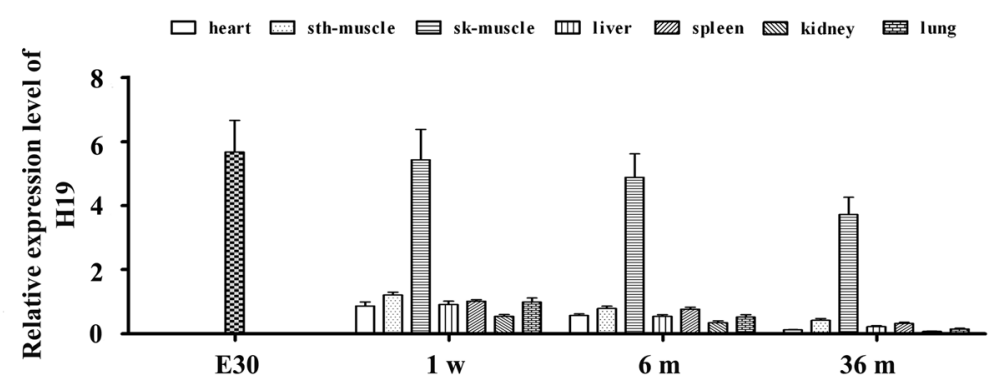

Fig. 1 The expression profiles of $\mathrm{H} 19$ in various tissues from male cattle of different postnatal stages. GAPDH RNA is the reference gene. The relative expression in satellite and $\mathrm{C}_{2} \mathrm{C}_{12}$ cells during differentiation was calculated according to the $2^{-\Delta \Delta C t}$ method. sth-muscle: smooth muscle; sk-muscle: tibia skeletal muscle; 1w: six 1-week old cattle, 1 m: 1-month old cattle, 6 m: 6-month old cattle, 36 m: 36-month old cattle. E30: samples from embryos after one month of development. The error bars were calculated with three repetitions 

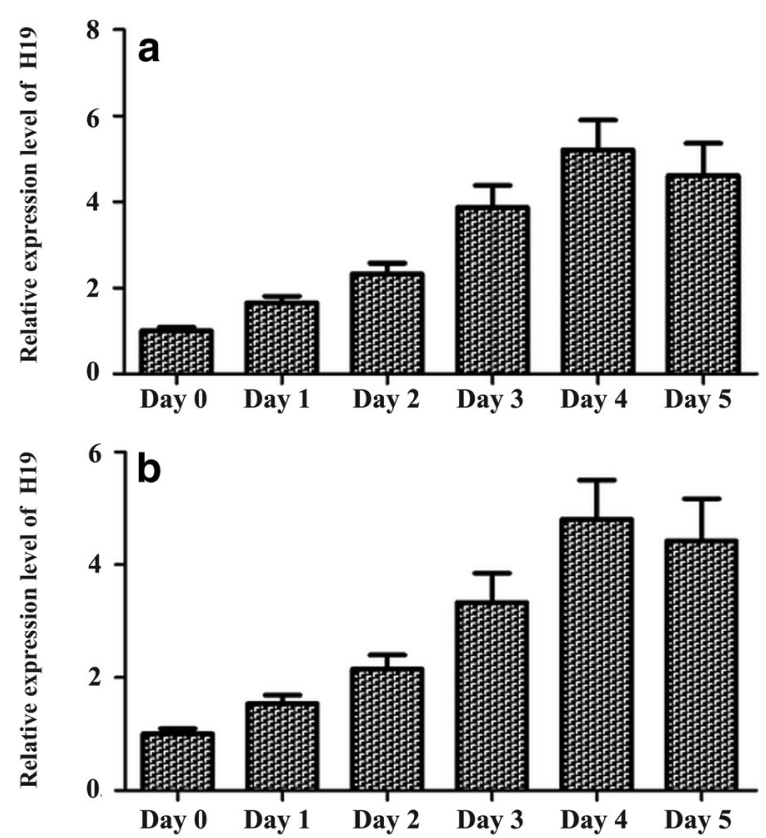

Fig. 2 Expression levels of $\mathrm{H} 19$ during differentiation of bovine tibia skeletal muscle (sk-muscle) satellite cells (a) and $\mathrm{C}_{2} \mathrm{C}_{12}$ myoblast cells (b)

of $\mathrm{H} 19$ are required in bovine myoblast differentiation and that its function might be achieved through Sirt1 and/or FoxO1 suppression.

\section{Overexpression of Sirt1 and FoxO1 neutralized the promotion of myoblast differentiation} by $\mathrm{H} 19$ overexpression

To verify the role of $\mathrm{H} 19$ in promoting myoblast differentiation through the suppression of Sirt1 and/or FoxO1, Sirt1 or FoxO1 expression vectors were co-transfected with pcDNA$\mathrm{H} 19$ to the satellite cells and $\mathrm{C}_{2} \mathrm{C}_{12}$ cells. $\mathrm{H} 19$ was more highly expressed after pcDNA-H19 transfection (Fig. 4a). Western blotting and RT-qPCR analysis revealed that the expression levels of MyoG and MyoHC increased, while Sirt1 and FoxO1 expression decreased in the satellite cells and $\mathrm{C}_{2} \mathrm{C}_{12}$ cells with pcDNA-H19 transfection. After co-transfection with pcDNA-Sirt1 or pcDNA-FoxO1, the expression levels of MyoG and MyoHC decreased, while those for Sirt1 and FoxO1 increased (Fig. 4b-i), implying that Sirt1 and/or FoxO1 neutralized the promotion of MyoG and MyHC by overexpression of $\mathrm{H} 19$.

\section{Discussions}

Here, the role of IncRNA H19 in myoblast differentiation was investigated in bovine skeletal muscle satellite cells. We found that $\mathrm{H} 19$ was more highly expressed in postnatal bovine skeletal muscle and revealed a novel mechanism of H19 in the promotion of myoblast differentiation. This mechanism may be associated with blocking of the Sirt1/ FoxO1 signaling pathway.

H19 was one of the earliest identified lncRNAs [16]. It is thus far known that it plays roles in multiple biological processes, including negative regulation of body weight and cell proliferation [17], and it may also be involved in the formations of many types of cancer [7, 18-20]. 


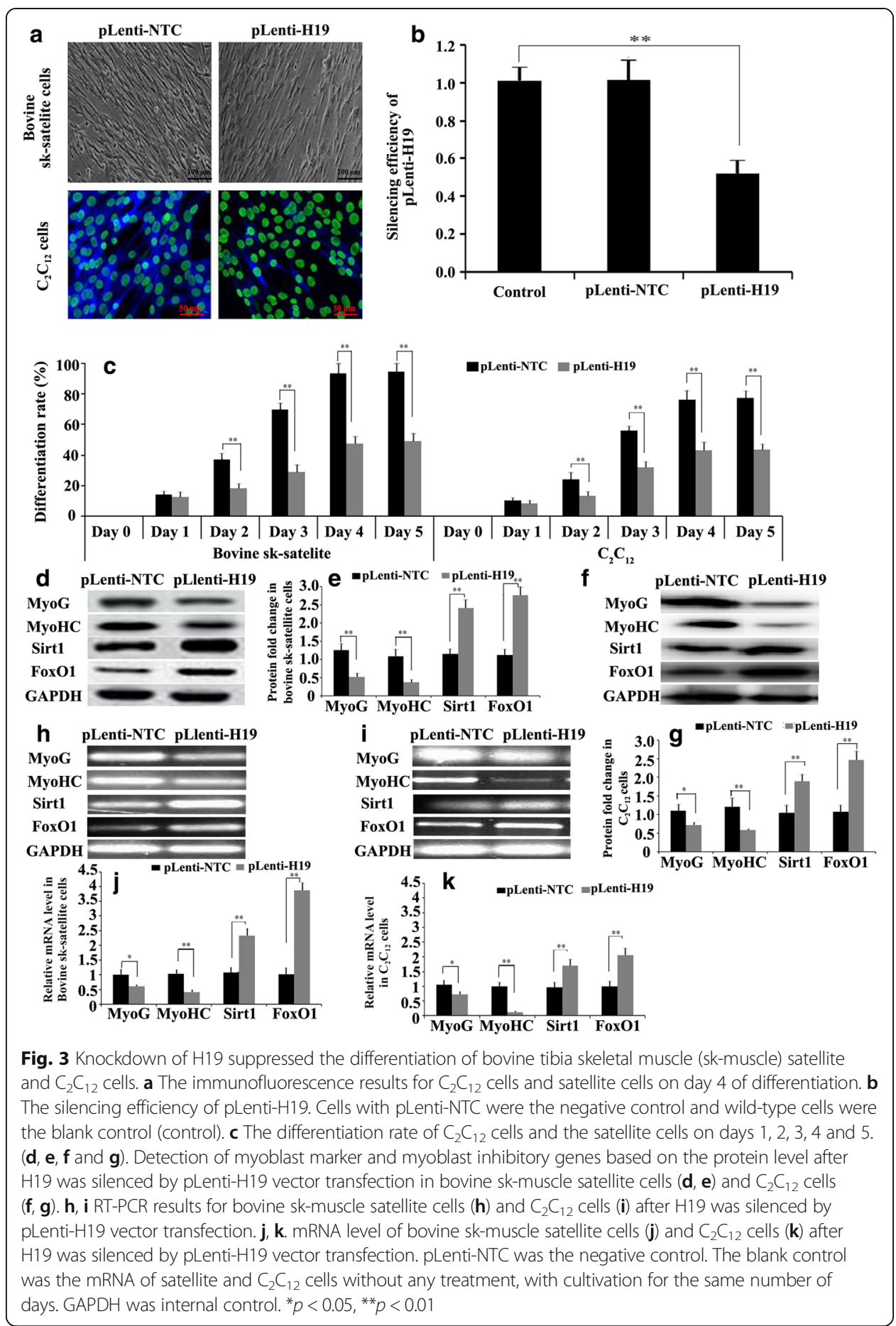

The roles and underlying mechanisms of H19 in the regulation of myogenesis or regeneration have recently been deeply investigated. H19 was shown to promote skeletal muscle differentiation through the Igf2 signaling pathway or miR-675-mediated gene suppression $[8,9]$. We found that the differentiation of bovine skeletal muscle satellite cells and $\mathrm{C}_{2} \mathrm{C}_{12}$ cells were suppressed (Fig. 3b) and that the expressions of Sirt1 and FoxO1 were enhanced after the silencing of $\mathrm{H} 19$ (Fig. 3d-k) but suppressed by its overexpression (Fig. 4). It was speculated that H19 promoted myoblast differentiation by 


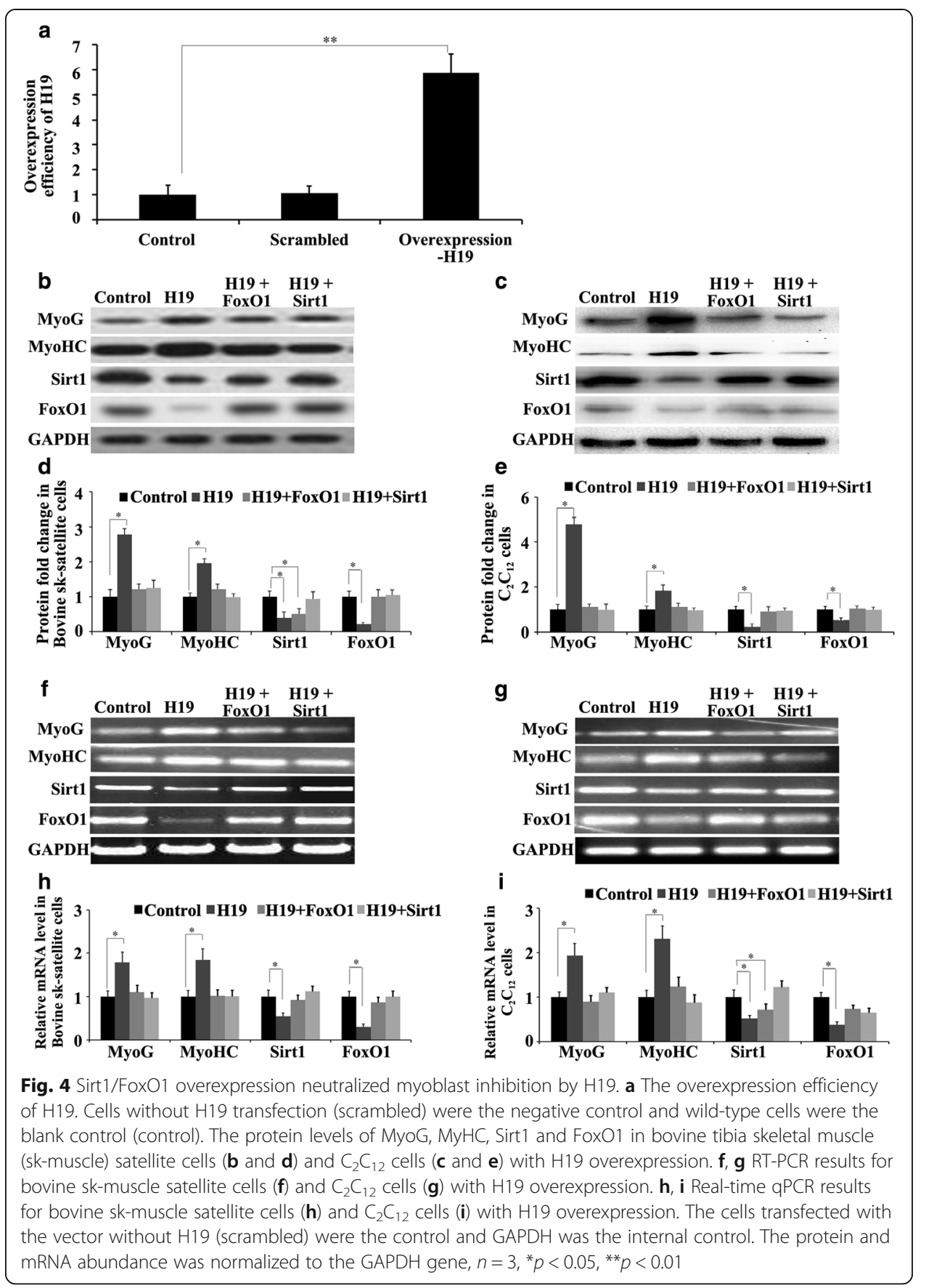

suppressing the Sirt1/FoxO1 signaling pathway. Further study is needed to investigate the underlying mechanism.

Based on their site of action and the level of gene expression, lncRNAs can be divided into functional categories, but since some lncRNAs plays distinct roles in multiple processes or the same role in dissimilar pathways, this distinction is not always easy or adequate. As an example, H19 has been verified to function positively in some cancers but negatively in other cancers [21-23], and in myoblast differentiation, H19 exhibits several mechanisms for the same promoting effect. 
In mammals, the majority of the transcriptional output is noncoding [24]. Compared to the well-studied coding genes, lncRNAs undoubtedly have greater research potential, especially in myogenesis.

\section{Conclusion}

$\mathrm{H} 19$ is required in the differentiation of bovine skeletal muscle satellite cells. its promoting effect might be associated with the blocking of the Sirt1/FoxO1 signaling pathway. Our findings revealed a novel pathway for H19 in the regulation of myogenesis.

\section{Additional file}

Additional file 1: Figure S1. The expression profiles of $\mathrm{H} 19$ in various tissues of cattle at different postnatal stages. The reference genes is $18 \mathrm{~S}$ RNA. The relative expression levels of $\mathrm{H} 19$ in satellite and $\mathrm{C}_{2} \mathrm{C}_{12}$ cells during differentiation were calculated according to the method of $2^{-\Delta \Delta C t}$. (TIF $498 \mathrm{~kb}$ )

\section{Abbreviations}

BSA: Bovine serum albumin; FoxO: Forkhead proteins; IncRNAs: Long noncoding RNAs; Myf5: Myogenic factor 5; MyoD: Myogenic differentiation antigen; PBS: Phosphate buffer solution; sk-muscle: Skeletal muscle; sth-muscle: Smooth muscle

\section{Acknowledgements}

The authors would like to thank professor Binyun Cao for his theoretical and technical contributions, and the Northwest Agriculture \& Forestry University of the Animal Academy of Sciences for the possibility to work on the experimental farm.

\section{Funding}

This research was supported by the National Key Research and Development Program of China (No. 2016YFD0500501), the National Science and Technology Support Program (No. 2012BAD39B01), the China Agriculture Research System (No. CARS-42) and the Agricultural Science and Technology Innovation Program (No. ASTIP-IAS07).

\section{Availability of data and materials}

Any additional information related with this study is available from the author for correspondence upon reasonable request.

\section{Authors' contributions}

Xiaochun $\mathrm{Xu}$ contribute to do the experiment for the revision of the manuscript, also contributed to the analysis and interpretation of data, as well as revision of the manuscript. Shengyue Ji contributed to the study design, acquisition, analysis and interpretation of data, and drafting and revision of the manuscript. Hongfu Zhang and Wenping Ma contributed to the study design and critical review of the manuscript. Weili Li, Bao Yi and Hengxin Li contributed to the cell culture and transfection, differentiation assay, RNA extraction, RT-PCR and real-time qPCR and western blotting. Hongfu Zhang contributed to getting financial support. All the authors read and approved the final manuscript.

\section{Competing interests}

The authors declare that they have no competing interests.

\section{Consent for publication}

Not applicable.

Ethics approval and consent to participate

Not applicable.

\section{Publisher's Note}

Springer Nature remains neutral with regard to jurisdictional claims in published maps and institutional affiliations.

Received: 20 October 2016 Accepted: 9 June 2017

Published online: 23 June 2017

\section{References}

1. Weintraub H. The MyoD family and myogenesis: redundancy, networks, and thresholds. Cell. 1993;75:1241-4.

2. Yusuf F, Brand-Saberi B. Myogenesis and muscle regeneration. Histochem Cell Biol. 2012;138:187-99.

3. Kitamura T, Kitamura Yl, Funahashi Y, et al. A Foxo/Notch pathway controls myogenic differentiation and fiber type specification. J Clin Invest. 2007;117:2477-85.

4. Batista PJ, Chang HY. Long noncoding RNAs: cellular address codes in development and disease. Cell. 2013;152: 1298-307. doi:10.1016/j.cell.2013.02.012. 
5. Heesch SV, Iterson MV, Jacobi J, et al. Extensive localization of long noncoding rnas to the cytosol and mono- and polyribosomal complexes. Genome Biol. 2014;15(1):1-12.

6. Cesana M, Cacchiarelli D, Legnini I, et al. A long noncoding RNA controls muscle differentiation by functioning as a competing endogenous RNA. Cell. 2011;147:358-69.

7. Gabory A, Jammes H, Dandolo L. The H19 locus: Role of an imprinted non-coding RNA in growth and development. Bioessays. 2010:32:473-80.

8. Dey BK, Pfeifer K, Dutta A. The H19 long noncoding RNA gives rise to microRNAs miR-675-3p and miR-675-5p to promote skeletal muscle differentiation and regeneration. Genes Dev. 2014:28:491-501.

9. Kallen AN, Zhou XB, Xu J, et al. The imprinted H19 IncRNA antagonizes let-7 microRNAs. Mol Cell. 2013;52:101-12.

10. Runge S, Nielsen FC, Nielsen J, et al. H19 RNA binds four molecules of insulin-like growth factor II mRNA-binding protein. J Biol Chem. 2000;275:29562-9.

11. Ciciliot S, Schiaffino S. Regeneration of mammalian skeletal muscle: basic mechanisms and clinical implications. Curr Pharm Des. 2010;16:906-14.

12. Kuang S, Gillespie MA, Rudnicki MA. Niche regulation of muscle satellite cell self-renewal and differentiation. Cell Stem Cell. 2008;2:22-31.

13. Kuang S, Rudnicki MA. The emerging biology of satellite cells and their therapeutic potential. Trends Mol Med. 2008;14:82-91

14. Schiaffino S, Partridge T. Skeletal muscle repair and regeneration, vol 3. In: Stienen GJM, Editor, Advances in Muscle Research. Dordrecht, Netherlands: Springer Press, 2008. p. 2002-2008.

15. Vann R, Althen T, Smith W, Veenhuizen J, Smith S. Recombinant bovine somatotropin (rbST) administration to creep-fed beef calves increases muscle mass but does not affect satellite cell number or concentration of myosin light chain-1f mRNA. J Anim Sci. 1998;76:1371-9.

16. Zhang Y, Tycko B. Monoallelic expression of the human H19 gene. Nat Genet. 1992;1:40-4.

17. Gabory A, Ripoche MA, Le Digarcher A, et al. $\mathrm{H} 19$ acts as a trans regulator of the imprinted gene network controlling growth in mice. Development. 2009;136:3413-21.

18. Cui H, Onyango P, Brandenburg S, et al. Loss of imprinting in colorectal cancer linked to hypomethylation of $\mathrm{H} 19$ and IGF2. Cancer Res. 2002;62:6442-6.

19. DeBaun MR, Niemitz EL, McNeil DE, et al. Epigenetic Alterations of H19 and LIT1 Distinguish Patients with Beckwith-Wiedemann Syndrome with Cancer and Birth Defects. Am J Hum Genet. 2002;70:604-11.

20. Hibi K, Nakamura H, Hirai A, et al. Loss of H19 imprinting in esophageal cancer. Cancer Res. 1996;56:480-2.

21. DeBaun MR, Niemitz EL, Feinberg AP. Association of In Vitro Fertilization with Beckwith-Wiedemann Syndrome and Epigenetic Alterations of LIT1 and H19. Am J Hum Genet. 2003;72:156-60.

22. Lottin $\mathrm{S}$, Adriaenssens $\mathrm{E}$, Dupressoir $\mathrm{T}$, et al. Overexpression of an ectopic $\mathrm{H} 19$ gene enhances the tumorigenic properties of breast cancer cells. Carcinogenesis. 2002;23:1885-95.

23. Matouk IJ, Mezan S, Mizrahi A, et al. The oncofetal H19 RNA connection: hypoxia, p53 and cancer. Biochim Biophys Acta Mol Cell Res. 2010;1803:443-51

24. Liu G, Mattick JS, Taft RJ. A meta-analysis of the genomic and transcriptomic composition of complex life. Cell Cycle. 2013;12:2061-72.

\section{Submit your next manuscript to BioMed Central and we will help you at every step:}

- We accept pre-submission inquiries

- Our selector tool helps you to find the most relevant journal

- We provide round the clock customer support

- Convenient online submission

- Thorough peer review

- Inclusion in PubMed and all major indexing services

- Maximum visibility for your research

Submit your manuscript at www biomedcentral.com/submit 